\title{
Coccidiose em cães e gatos do município de Andradina, estado de São Paulo, Brasil
}

\author{
Coccidiosisin dogs and cats at the Municipality of Andradina in the State of São Paulo, \\ Brazil
}

\section{Willian Marinho Dourado COELHO ${ }^{1}$; Alessandro Francisco Talamini do AMARANTE'; Silvia Helena Venturoli PERRI ${ }^{3}$; Natalia Marinho Dourado COELHO ${ }^{3}$; Juliana de Carvalho APOLINÁRIO ${ }^{4}$; Weslen Fabricio Pires TEIXEIRA ${ }^{3}$; Katia Denise Saraiva BRESCIANI ${ }^{3}$}

\author{
${ }^{1}$ Departamento de Medicina Veterinária Preventiva da Faculdade de Ciências Agrárias e Veterinárias, Universidade Estadual \\ Paulista, Jaboticabal - SP, Brasil \\ ${ }^{2}$ Departamento de Parasitologia do Instituto de Biociências, Universidade Estadual Paulista, Botucatu - SP, Brasil \\ ${ }^{3}$ Departamento de Apoio, Produção e Saúde Animal da Faculdade de Medicina Veterinária da Universidade Estadual Paulista, \\ Araçatuba - SP, Brasil \\ ${ }^{4}$ Programa Multicêntrico de Pós-Graduação em Ciências Fisiológicas da Faculdade de Medicina Veterinária daUniversidade \\ Estadual Paulista, Araçatuba - SP, Brasil
}

\begin{abstract}
Resumo
A coccidiose é uma das mais frequentes enfermidades parasitárias em pequenos animais em todo o mundo. O objetivo deste trabalho foi determinar a ocorrência da infecção por Cystoisospora em cães e gatos no Município de Andradina, São Paulo. Durante os anos de 2007 a 2009, amostras fecais de 97 gatos e 93 cães foram processadas por meio das técnicas de flutuação em solução saturada de cloreto de sódio e sedimentação espontânea. As espécies foram identificadas por morfometria dos oocistos. Oocistos fecais de Cystoisospora foram encontrados em 71,1\% (69/97) dos gatos, sendo que infecção simples por C. rivoltaou por C. felis ocorreu, respectivamente, em 41,0\% (16/39) e em 20,5\% (8/39) dos animais, com $\mathrm{P} \geq 0,2319$. Em 39,7\% (37/93) dos cães foi detectada positividade para Cystoisospora spp., sendo a espécie C. canis identificada na maior proporção $(63,9 \%)$ com $\mathrm{P}=0,0005$. A partir dos resultados obtidos, nós concluímos que cães e gatos tiveram elevada ocorrência de infecção por Cystoisospora, sendo C. canis e C. rivolta as espécies mais observadas, respectivamente.
\end{abstract}

Palavras-chave: Coccidios. Cystoisosporinae. Infecção natural. Animais de Companhia.

\begin{abstract}
Coccidiosis is one of the most common parasitic diseases in dogs and cats in all the world. The aim of this study was to determine the occurrence of this parasitosis in dog and cat population at the Municipality of Andradina in the State of São Paulo, from 2007 to 2009. Fecal samples from 97 cats and 93 dogs were analyzed by using the techniques of flotation in saturated sodium chloride and spontaneous sedimentation. The species were classified according to morphology of the oocysts. Cystoisospora fecal oocyst found in $71.1 \%(69 / 97)$ of the cats, and simple infection by C. rivolta and C. felis occurred respectively in $41.0 \%(16 / 39)$ and $20.5 \%(8 / 39)$ animals, with $\mathrm{P} \geq 0.2319$. In $39.7 \%(37 / 93)$ of the dogs was found positive for Cystoisospora spp. And the species C. canis identified in the largest proportion $(63.9 \%)$ with $\mathrm{P}=$ 0.0005. From the results, we conclude that dogs and cats had high incidence of infection Cystoisospora, being C. canis and C. rivolta most observed species, respectively.
\end{abstract}

Keywords: Coccidia. Cystoisosporinae. Natural Infection. Companion animals.

\section{Introdução}

O gênero Cystoisospora ocasiona diarreia em pequenos animais, com elevado número de casos clínicos ${ }^{1,2}$ e apesar disso, são poucos os pesquisadores que se dedicam a seu estudo, no Brasil ${ }^{3,4,5}$ e no mundo, como na Grécia ${ }^{6}$, Holanda $^{7}$, Venezuela ${ }^{8}$, Emirados Árabes ${ }^{9}$, Russia $^{10}$, Austrália ${ }^{11}$, Estados Unidos da América ${ }^{12,13}$ e no $\operatorname{Iran}^{14}$.
Correspondência para:

Willian Marinho Dourado COELHO

Universidade Estadual Paulista, Faculdade de Ciências Agrárias e

Veterinárias, UNESP

Departamento de Medicina Veterinária Preventiva

Via de acesso prof. Paulo Donatto Castellani, s/n

CEP: 14884-900, Jaboticabal, São Paulo, Brasil

e-mail - willianmarinho@hotmail.com

Recebido: 05/10/2010

Aprovado: 18/04/2012 
No cão, a infecção se dá principalmente por C. canis, C. ohioensis, C. burrowsie C. neorivolta ${ }^{15,16,17,18}$, enquanto que, nos felinos, por C. felise C. rivolta $a^{19,20,21,22}$.

O objetivo deste trabalho foi determinar a ocorrência da infecção por Cystoisospora em cães e gatos do município de Andradina, São Paulo, Brasil.

\section{Material e Método}

O estudo foi conduzido entre março de 2007 e novembro de 2009, no município de Andradina no estado de São Paulo, Brasil. Foram utilizados animais procedentes do Centro de Controle de Zoonoses do referido município, com aprovação prévia pelo Comitê de ética em Experimentação Animal (FOAAraçatuba), protocolo 2007-003276. Dos 93 cães, 45 eram fêmeas e 48 machos, 47 tinham raça definida (RD) e 46 eram sem raça definida (SRD), sendo 86 oriundos da área urbana e sete da rural, como também, 15 eram jovens, 55 adultos e 23 idosos. Entre os 97 gatos, 59 eram fêmeas e 38 machos, com 13 animais RD e 84 SRD, 73 da zona urbana e 24 da rural, sendo que 32 eram jovens, 51 eram adultos e 14 eram idosos.
Os animais foram contidos fisicamente e, por meio de estimulação anal, eram as fezes colhidas diretamente do reto, numa quantidade mínima de dois gramas e acondicionadas em potes coletores estéreis. Todas as amostras fecais foram analisadas pelas técnicas de flutuação em solução saturada de cloreto de sódio ${ }^{23}$ e sedimentação simples ${ }^{24}$, sendo as espécies classificadas de acordo com a morfometria dos oocistos conforme proposto por Dubey et al. ${ }^{1}$ e Smith ${ }^{25}$ utilizandose ocular de campo amplo micrométrica 10x (Bioval $\left.{ }^{\circ}\right)$ com objetiva de 40x.

Avaliou-se a influência da idade, sexo, raça e origem dos cães e gatos na ocorrência das espécies de Cystoisospora bem como a relação entre a alteração na consistência das fezes e a presença dos parasitos. Os dados foram analisados pelo teste qui-quadrado $\left(\chi^{2}\right)$ e pelo teste exato de Fisher.

\section{Resultados}

Nos felinos, a ocorrência de oocistos do gênero Cystoisospora foi de 71,1\% (69/97) e não foi correlacionada com as variáveis raça, sexo, idade, área e consistência fecal das amostras (Tabela 1). A infecção simples

Tabela 1 - Ocorrência da infecção por Cystoisospora spp. por meio das técnicas de acordo com a raça, sexo, idade, procedência e consistência fecal de 93 cães e 97 gatos

\begin{tabular}{|c|c|c|c|c|c|c|c|c|c|}
\hline \multirow{2}{*}{ Variável } & \multirow{2}{*}{ Categoria } & \multicolumn{4}{|c|}{ Ocorrência em cães } & \multicolumn{4}{|c|}{ Ocorrência em gatos } \\
\hline & & $\mathrm{n}$ & Positivo & $\%$ & $\mathrm{P}$ & $\mathrm{n}$ & Positivo & $\%$ & $\mathrm{P}$ \\
\hline \multirow{2}{*}{ Raça } & $\mathrm{RD}$ & 47 & 14 & 29,8 & \multirow{2}{*}{$0,1158(1)$} & 13 & 6 & 46,2 & \multirow{2}{*}{$0,1713(1)$} \\
\hline & SRD & 46 & 22 & 47,8 & & 84 & 33 & 39,3 & \\
\hline \multirow{2}{*}{ Sexo } & Fêmea & 45 & 13 & 28,9 & \multirow{2}{*}{$0,0950(1)$} & 59 & 23 & 39,0 & \multirow{2}{*}{$0,9251(1)$} \\
\hline & Macho & 48 & 23 & 47,9 & & 38 & 16 & 42,1 & \\
\hline \multirow{2}{*}{ Idade } & Jovem & 70 & 30 & 42,9 & \multirow{2}{*}{$0,2357(1)$} & 73 & 26 & 35,6 & \multirow{2}{*}{$0,8681(1)$} \\
\hline & Adulto & 23 & 6 & 26,1 & & 24 & 13 & 54,2 & \\
\hline \multirow{2}{*}{ Área } & Urbana & 86 & 29 & 33,7 & \multirow{2}{*}{$0,0009(2)$} & 73 & 27 & 27,0 & \multirow{2}{*}{$0,3745(1)$} \\
\hline & Rural & 7 & 7 & 100,0 & & 24 & 12 & 50,0 & \\
\hline \multirow{4}{*}{$\begin{array}{l}\text { Consistência } \\
\text { Fecal }\end{array}$} & Liquefeita & 18 & 13 & 72,2 & \multirow{4}{*}{$<0,0001(1)$} & 15 & 4 & 26,7 & \multirow{4}{*}{$0,6866(1)$} \\
\hline & Pastosa & 29 & 15 & 51,7 & & 32 & 13 & 40,6 & \\
\hline & Semi-sólida & 27 & 7 & 25,9 & & 12 & 5 & 41,7 & \\
\hline & Sólida & 17 & 1 & 5,9 & & 38 & 17 & 44,7 & \\
\hline
\end{tabular}

(1) teste $\chi^{2(2)}$ teste exato de Fisher 
por C. rivolta ou por C. felis ocorreu, respectivamente, em $41,0 \%(16 / 39)$ e em $20,5 \%$ (8/39) dos animais e infecção mista pelas duas espécies supramencionadas em 38,5\% (15/97), com $P \geq 0,2319$. Também não foi evidenciada associação entre a presença de uma determinada espécie de Cystoisopora e alterações na consistência das fezes ( $\mathrm{P} \geq 0,1587)$, como pode ser evidenciado na tabela 2.

Ocorrência da infecção por Cystoisospora foi de $39,7 \%$ (37/93) nos cães e não foi associada a padrão racial $(P \geq 0,1158)$, sexo $(P \geq 0,0950)$ ou faixa etária $(\mathrm{P} \geq 0,2357)$. Todos os animais examinados na área rural foram positivos, com presença do protozoário em 100\% (7/7) dos mesmos ( $\mathrm{P}=0,0009)$. Adicionalmente, houve porcentagem de positividade de $72,2 \%$, $51,7 \%, 25,9 \%$ e 5,9\%, respectivamente, para amostras com consistência fecal liquefeita, pastosa, semi-sólida e sólida, com $\mathrm{P} \leq 0,0001$ (Tabela 1 ). A espécie $C$. canis foi identificada na maior proporção $(63,9 \%)$ nos animais do estudo $(\mathrm{P}=0,0005)$, mas não foi constatada correlação da espécie de coccídio com estado físico das amostras fecais (Tabela 2).

\section{Discussão}

O gênero Cystoisospora foi constatado em 71,1\% (69/97) dos gatos examinados. Resultados inferiores aos encontrados em nosso estudo, foram verificados no Brasil, por Lorenzini, Tasca e De Carli ${ }^{4}$ em Por- to Alegre, Rio Grande do Sul e no estado do Rio de Janeiro por Nery-Guimarães e Lage ${ }^{26}$. Divergências nos resultados obtidos nos diversos trabalhos podem ser justificadas, em parte, por serem utilizadas diferentes técnicas coproparasitológicas na execução destas pesquisas.

Em nosso trabalho, não evidenciamos correlação desta coccidiose com a faixa etária dos felinos em questão, diferindo dos achados de Lorenzini, Tasca e De Carli ${ }^{4}$ que evidenciaram maior incidência, de zero a seis, entre 13 a 24 e maior que 49 meses de idade.

Nos felinos examinados, registrou-se maior ocorrência de C. rivolta. No entanto, outros autores na literatura demonstraram predominância de $C$. felis em relação ao primeiro ${ }^{9,11,26}$, sugerindo influência do local de origem dos animais sobre a prevalência destes protozoários.

Em nosso trabalho, observamos que 39,7\% dos cães eram hospedeiros definitivos de Cystoisospora. Interessante notar que esta porcentagem de positividade está acima do esperado, se compararmos os resultados de outros pesquisadores nos EUA $^{13}$, Terra Nova $^{27}$ e em outras regiões do Brasil ${ }^{28,29}$. A idade de até seis meses foi apontada como um fator predisponente por Little et al. ${ }^{13}$, o que não foi confirmado no presente trabalho.

Todos os cães analisados neste experimento, provenientes de áreas rurais foram positivos para a esta protozoose. No entanto, deve-se atentar para o detalhe de que foram examinados sete animais e esta quantidade

Tabela2 - Ocorrência de espécies do gênero Cystoisospora spp. em relação à consistência fecal de 36 cães e 39 gatos positivos

\begin{tabular}{|c|c|c|c|c|c|c|c|c|c|c|}
\hline \multirow{3}{*}{ Hospedeiro } & \multirow{3}{*}{ Espécie de Isospora } & \multicolumn{8}{|c|}{ Consistência fecal } & \multirow{3}{*}{$\mathrm{P}(1)$} \\
\hline & & \multicolumn{2}{|c|}{ Liquefeita } & \multicolumn{2}{|c|}{ Pastosa } & \multicolumn{2}{|c|}{ Semi-sólida } & \multicolumn{2}{|c|}{ Sólida } & \\
\hline & & $\mathrm{n}$ & $\%$ & $\mathrm{n}$ & $\%$ & $\%$ & & $\mathrm{n}$ & $\%$ & \\
\hline \multirow{3}{*}{ Cão } & C. canis & 10 & 27,8 & 7 & 19,4 & 6 & 16,7 & - & - & \\
\hline & C. ohioenis & 2 & 5,6 & 3 & 8,3 & - & - & 1 & 2,8 & 0,1613 \\
\hline & C. canis + C. ohiensis & 1 & 2,8 & 5 & 13,9 & 1 & 2,8 & - & - & \\
\hline \multirow{3}{*}{ Gato } & C. rivolta & 2 & 5,1 & 4 & 10,3 & 1 & 2,6 & 9 & 23,1 & \\
\hline & C. felis & - & - & 1 & 2,6 & 2 & 5,1 & 5 & 12,8 & 0,1587 \\
\hline & C. rivolta + C. felis & 2 & 5,1 & 8 & 20,5 & 2 & 5,1 & 3 & 7,7 & \\
\hline
\end{tabular}

(1) teste exato de Fisher 
diminuta, de certa forma, impede que afirmemos categoricamente que a origem é fator predisponente para a ocorrência da referida parasitose. Quanto ao local de origem dos animais, Dubná et al. ${ }^{30}$ notificaram elevada taxa de infecção, principalmente naqueles que permaneceram por longos períodos em abrigos.

\section{Referências}

1.DUBEY, J. P. The evolution of the knowledge of cat and dog coccidia. Parasitology, v. 136, n. 12, p. 1469-75, 2009.

2.DUBEY, J. P.; LINDSAY, D. S.; LAPPIN, M. R. Toxoplasmosis and other intestinal coccidial infections in cats and dogs. Veterinary Clinics of North America: small animal practice, v. 39, n. 6, p. 1009-1034. 2009.

3. COELHO, W. M. D.; AMARANTE, A. F. T. do.; SOUTELLO, R. V. G.; MEIRELES, M. V.; BRESCIANI, K. D. S. Ocorrência de parasitos gastrintestinais em amostras fecais de felinos no município de Andradina, São Paulo. Revista Brasileira de Parasitologia Veterinária, v. 18, n. 2, p. 46-49, 2009.

4. LORENZINI, G.; TASCA, T.; DE CARLI, G. A. Prevalence of intestinal parasites in dogs and cats under veterinary care in Porto Alegre, Rio Grande do Sul, Brazil. Brazilian Journal of Veterinary Research and Animal Science, v. 44, n. 2, p. 137 $145,2007$.

5.RAGOZO, A. M. A.; MURADIAN, V.; RAMOS E SILVA, J. C.; RENATO CARAVIERI, R.; AMAJONER, V. R. MAGNABOSCO, C.; SOLANGE MARIA GENNARI, S. M. Ocorrência de parasitas gastrintestinais em fezes de gatos da cidade de São Paulo e Guarulhos. Brazilian Journal of Veterinary Research and Animal Science, v. 39, n. 5, p. 244246, 2002.

6. PAPAZAHARIADOU, M.; FOUNTA, A.; PAPADOPOULOS, E.; CHLIOUNAKIS, S.; ANTONIADOU-SOTIRIADOU, K.; THEODORIDES, Y. Gastrointestinal parasites of shepherd and hunting dogs in the Serres Prefecture, Northern Greece. Veterinary Parasitology, v. 148, n. 2, p. 170-173, 2007.

7. ROBBEN, S. R.; LE NOBEL, W. E.; DÖPFER, D.; HENDRIKX W. M.; BOERSEMA, J. H.; FRANSEN, F.; EYSKER, M. E. Infection with helminths and/or protozoa in cats in animal shelthers in the Netherlands. Tjidschrift Voor Diergeeneskunde, v. 129, n. 1, p. 2-6, 2004.

8. RAMÍREZ-BARRIOS R. A.; BARBOZA-MENA, G.; MUÑOZ, J.; ANGULO-CUBILLÁN, F; HERNÁNDEZ, E.; GONZÁLEZ, F.; ESCALONA, F. Prevalence of intestinal parasites in dogs under veterinary care in Maracaibo, Venezuela. Veterinary Parasitology, v. 121, n. 1-2, p. 11-20, 2004.

9.SCHUSTER, R. K.; THOMAS, K.; SIVAKUMAR, S.; O'DONOVAN, D. The parasite fauna of stray domestic cat (Feliscatus) in Dubai, United Arab Emirates. Parasitology Research, v. 105, n. 1, p. 125-34, 2009.

10.SERGIEV, V. P.; USPENSKÍÍ, A. V.; GOROKHOV, V. V. The current canine and feline parasitic diseases situation in the megapolis of Moscow. Meditsinskaia Parazitologiia I Parazitarnye Bolezni, n. 1, p. 17-20, 2007.

11.PALMER, C. S.; THOMPSON, R. C.; TRAUB, R. J.; REES, R.; ROBERTSON, I. D. National study of the gastrointestinal parasites of dogs and cats in Australia. Veterinary Parasitology, v. 15 , n. 1, p. 181-190, 2008.

\section{Conclusões}

A partir dos resultados obtidos, pode ser concluído que cães e gatos no referido município apresentaram elevada ocorrência de infecção por Cystoisospora, sendo C. canis e C. rivolta as espécies mais observadas em ambas populações, respectivamente.

12.GATES, M. C.; NOLAN, T. J. Endoparasite prevalence and recurrence across different age groups of dogs and cats. Veterinary Parasitology, v. 166, n. 1-2, p. 153-158, 2009.

13.LITTLE, S. E.; JOHNSON, E. M.; LEWIS, D.; JAKLITSCH, R. P.; PAYTON, M. E.; BLAGBURN, B. L.; BOWMAN, D. D.; MOROFF, S.; TAMS, T.; RICH, L.; AUCOIN, D. Prevalence of intestinal parasites in pet dogs in the United States. Veterinary Parasitology, v. 166, n. 1-2, p. 144-1452, 2009.

14.MIRZAEI, M. Prevalence of stray dogs with intestinal protozoan parasites. American Journal of Animal and Veterinary Sciences, v. 5, n. 2, p. 79-83, 2010.

15.BUEHL, I. E.; PROSL, H.; MUNDT, H. C.; JOACHIM, A. Canine isosporosis: epidemiology of field and experimental infections. Journal of Veterinary Medicine, v. 53, n. 10, p. 482-487, 2006.

16.DUBEY, J. P. Isosporaohioensis sp. n. proposed for I. rivolta of the dog. The Journal of Parasitology, v. 61, n. 3, p. 462-465, 1975.

17.DUBEY, J. P.; MAHRT, J. L. Isosporaneorivolta sp. n. from the domestic dog. The Journal of Parasitology, v. 64, n. 6, p. 10671073, 1978.

18.TRAYSER, C. V.; TODD JR. K. S. Life cycle of Isosporaburrowsi n. sp. (Protozoa: Eimeriidae) from the dog Canisfamiliaris. American Journal of Veterinary Research, v. 39, n. 1, p. 9598, 1978.

19.DUBEY, J. P. Life cycle of Isosporarivolta (Grassi, 1879) in cats and mice. The Journal of Protozoology, v. 26, n. 3, p. 433-443, 1979.

20.LINDSAY, D. S.; DUBEY, J. P.; BLAGBURN, B. Biology of Isospora spp. from human, nonhuman primates, and domestic animals. Clinical Microbiology Reviews, v. 10, n. 1, p. 19-34, 1997.

21.WENYON, C. M. Coccidiosis of cats and dogs and the status of the Isospora of man. Annals of Tropical Medicine and Parasitology, v. 17, p. 231-288, 1923.

22.WENYON, C. M. Coccidia of the genus Isosporain cats, dogs, and man. Parasitology, v. 18, n. 2, p. 253-266, 1926.

23.WILLIS, H. H. A simple levitation method for the detection of hookworm ova. The Medical Journal of Australia, v. 8, p. 375-376, 1921.

24.SLOSS, M. W.; ZAJAC, A. N.; KEMP, R. L. Parasitologia clínica veterinária. São Paulo: Manole, 1999. 198 p.

25.SMITH, D. D. The Sarcocystidae: Sarcocystis, Frenkelia, Toxoplasma, Besnoitia, Hammondia and Cystoisospora. Journal of Protozoology, v. 28, n. 2, p. 262-266, 1981.

26.NERY-GUIMARÃES, F; LAGE, H. A. Prevalência e ciclo evolutivo de "Isosporafelis" Wenyon, 1923 e "Isosporarivolta" (Grassi, 1879) Wenyon, 1923 em gatos. Memórias do Instituto 
Oswaldo Cruz, v. 71, n. 1-2, p. 43-54, 1973.

27.BRIDGER, K. E.; WHITNEY, H. Gastrointestinal parasites in dogs from the Island of St. Pierre off the south coast of Newfoundland. Veterinary Parasitology, v. 162, n. 1-2, p. 167170, 2009.

28. OLIVEIRA-SEQUEIRA, T. C. G.; AMARENTE, A. F.; FERRARI, T. B.; NUNES, L. C. Prevalence of intestinal parasites in dogs from São Paulo State, Brazil. Veterinary Parasitology, v. 103, n. 1-2, p. 103-119, 2002.
29.KATAGIRI, S.; OLIVEIRA-SEQUEIRA, T. C. Prevalence of dog intestinal parasites and risk perception of zoonotic infection by dog owners in São Paulo State, Brazil. Zoonosis and Public Health, v. 55, n. 8-10, p. 406-413, 2008.

30.DUBNÁ, S.; LANGROVÁ, I.; NÁPRAVNÍK, J.; JANKOVSKÁ, I.; VADLEJCH, J.; PEKÁR, S.; FECHTNER, J. The prevalence of intestinal parasites in dogs from Prague, rural areas and shelters of the Czech Republic. Veterinary Parasitology, v. 145, n. 1-2, p. 120-128, 2007. 